\title{
Observational Constraints on Cosmological Superstrings
}

\author{
Olga S. Sazhina ${ }^{1 *}$ and Alfiia I. Mukhaeva ${ }^{2}$ \\ ${ }^{1}$ Sternberg State Astronomical Institute of Lomonosov Moscow State University, Moscow, Russian Federation \\ ${ }^{2}$ Dubna State University, Dubna, Russian Federation \\ Email: cosmologia@yandex.ru
}

\begin{abstract}
The existance of cosmic strings does not contradict to the current observational cosmological data. From theoretical point of view the cosmic strings can be of the different origins and type and be characterized by wide range of energies. The cosmic superstrings naturally arise in the brane-world scenario. The paper is devoted to discussing possible cosmological observational tests on superstring theory, and to the identification of observational properties allowing to distinguish between cosmological superstring of different type. In the paper we obtained a lower limit on the superstring tension as function of the deficit angle.
\end{abstract}

Keywords: Cosmic strings, cosmological FD-strings, CMB anisotropy

\section{Introduction}

Cosmic strings are one-dimensional objects of cosmological scales (see [1], [2], [3], [4], [5], [6], [7] and the refs. therein). In the simplest models of a single straight string with one scalar field, the string has no ends. Therefore, its length is limited by the surface of the last scattering. The more far the string from the observer, so it is shorter. Consequently, the size of the string is limited to the diameter of the surface of the last scattering and can be arbitrary. These exotic structures are predicted by theory, but have not been found yet. The strings can be responsable for no more than $4.4 \%$ of the CMB anisotropy signal (at multipole $l=10)$, [8].

Cosmic strings may be purely topological entities (endless and in the form of closed loops), formed as a result of phase transitions in the vacuum stages of the expansion and cooling of the early universe, or hybrid topological and field configurations (for example, a string with monopols on its end and conglomerates of such elements, so-called "necklaces"), [9].

The theory of superstrings allows the formation of cosmological superstrings, at least in the braneworld scenario (interactions among multidimensional spaces), [10]. Defects of this type include coherent macroscopic states of fundamental superstrings (F-strings), and Dp-branes extended in one macroscopic direction (we will use the designations "D-branes"and "D-strings"to avoid confusion between the number of compacted dimension in Dp-branes and p-number of F-strings), [11], [12], [13].

Search for cosmological superstrings by astrophysical methods requires preliminary assessment of the properties of possible observable candidates on cosmic strings, namely, upper limits on cosmic string linear density.

There are two main groups of radio methods to find such limits.

- String network simulations (see [3] and refs. therein) and search for cumulative restriction for string angular power spectrum in CMB data, [8];

- Direct search for individual strings through step-like temperature discontinuity, [14] (statement of Canny algorithm for future CMB experiments), [15] (method based on Haar convolution, i.e. MHF method), where the search for temperature gradients in CMB maps is discussed.

The first group of the methods give an upper limit to estimate the fraction of energy in string with respect to the total energy of the Universe. According to the current observational data, [16], there is no reason to assume that the strings have to manifest themselves in the form of network only. The negative results in the optical data to analyse the gravitational lens statistics indicate that (if the strings exist) they should be in small amount, [17]. Thus, the technique to recognize a single string and evaluate its possible features becomes relevant. 
Both methods are based on the analysis of the CMB anisotropy. The knowledge of the anisotropy induced by a string establishes a relationship between string velocity and string linear density. The linear density has to completely define the conical geometry of string space-time. On the other hand the string linear density is related with the string deficit angle (the cone angle). The last one can be estimated independently by method of gravitational lensing.

In this work we used our previous results in cosmic string search combining the radio and optical methods, [18], [15].

Let us describe briefly these results. We searched for the special temperature jumps in CMB anisotropy radio data. These step-like jumps indicate positions of string candidates. So, we established a location of several string candidates. We chose the most promising one to continue a verification of this object as a string by other methods. We checked it by optical surveys looking for the chains of particular gravitational lensing events (in preparation). We found a few dozen of such lens candidates. We estimated the mean deficit angle, measuring the apparent angular separation between images produced by lensing of galaxies by the string.

Therefore, as "input"for the present paper we have two observational properties of a string: (a) the amplitude of the string anisotropy $\delta T / T$ and (b) mean deficit angle $\Delta \Theta$ of this string. Of course we always have to speak about the "lens candidate"and the "string candidate"even if we have the strong enough evidences to its existence.

The aim of this paper is to demonstrate a relationship between the observable properties of a string with parameters of cosmological superstring, to find the lower observational limit on superstring linear density in brane-world model.

In addition to the two techniques (CMB anisotropy and gravitational lensing), there are several model dependent methods of cosmic string search. So, cosmic strings can manifest themselves as sources of gamma rays, and also can have a wide range of the folloving astrophysical signatures: synchrotron radiation, radio bursts, ultra high energy cosmic rays and effects from stochastic gravitational wave background, [19], [20], [21]. The last one becomes promising but is very sensitive to the nature of the string evolution and is intended to analize the string networks (a gravitational field of a single string will not equally affect all pulsars).

The paper is organized as follows. In the Section 2 the status of superstrings in modern cosmology is briefly described. In the Section 3 the warp factor is considered as a parameter which could be in principle estimated by the known observable string properties (string deficit angle). In the fourth (the main) Section it is discussed the connection between 4D cosmological superstrings and 10D superstrings from observational point of view.

\section{Superstrings as Possible Cosmological Objects}

Theoretical studies of cosmic strings become more popular in modern cosmology. Classical topological strings are of great interest for understanding the processes of phase transitions of the vacuum in the early universe, to study the relic dark energy, and to study the symmetry violation of fundamental interactions. Cosmic strings could serve as unique indicators of composition and topological properties of post-inflationary epochs.

For the sake of completeness, we would like to add that the dark energy problem can be, in principle, achieved through the exteded theories of gravity, [22].

The existence of cosmic strings of different types, properties and origins is not in contradiction with the modern observational limits on CMB anisotropy.

Hybrid cosmic string models become of particular interest because they are preferred both in terms of simulations [9], and from the point of view of the observational data on the CMB anisotropy (the most high upper limit from the analysis of the CMB angular spectra, [8], and from the search of individual strings, [15]). Interest in the cosmic strings is manifested by the superstring physics as such objects may be the only observational evidence for superstring models [23].

Firstly superstrings have been considered as possible cosmological objects since their energy can be significantly lower than the Planck one (about $10^{19} \mathrm{GeV}$ ). Thus, the tension of the cosmological superstrings would be comparable with the observational limits. This approach is implemented in the 
representation of brane-world (in a multi-dimensional space-time when additional spatial dimensions have certain properties). In the 4D space-time fundamental superstring can not be stretched to the cosmological scale, they "tear"and will become a system of microscopic superstrings. In the brane-world scenario an extra dimension reduction provides an existence of superstrings on the cosmological scales. Let us consider the cosmological model of type II of superstrings, the existing 10D space-time (the existence of other types of cosmological superstrings is likely in contradiction with the observational data; see [23] and refs. therein). In string theory the superstrings are not the only size-localized objects.

The theory admits the existence of a two-dimensional membrane or its analog of higher dimensions, called brane with various dimension; so the particle is a 0-brane, the string is 1-brane, etc. In the brane-world model it is introduced the concept of D-branes, which means that the brane satisfies the initial conditions of Dirichlet-type to fix the ends (the same way as it is done in the solution of differential equations in partial derivatives). Thus in the multi-dimensional space-time there are fundamental closed strings (loops), and fundamental strings which are ended in the D-branes. The model also allows anti-Dbranes (D- $\bar{D}$-branes): a brane and an anti-brane have equal and opposite charges, so they are attracted to each other. More generally, M-theory, too, contains the brane-world scenario. The common property of these models is that ordinary matter is concentrated on the hyper surface, the brane immersed in a space-time of higher dimension (called the bulk). Our universe may be one of such a brane. Gravity can be spread in the bulk (for review, see [24]).

Thus the superstring can naturally appear in brane-world scenario with energies that are comparable with observational constraints on cosmic string energies.

\section{Warp Factor in the 10D Space-time}

One of the main motivations of brane-world scenario was to explain the large energy gap between Planck scale of gravity $10^{19} \mathrm{GeV}$ and electroweak interactions, $10^{2} \mathrm{GeV}$. The model bases on the warp factor of the space-time. As in the general relativity the interval is defined

$$
d s^{2}=d t^{2}-d x^{2}
$$

in the warp space-time it is introduced the modified interval

$$
d s^{2}=e^{-A(y)}\left(d t^{2}-d x^{2}\right)-d y^{2} .
$$

In (1) - (2) the $x$ is a three-dimensional spatial vector, and in (2) the $y$ is coordinate (or coordinates) of additional compact dimension, $e^{-A(y)}$ is a positive function of the extra dimension, called warp factor (the function $A(y)>0$ ). The physical meaning of this function is that it is the gravitational redshift in a compact extra dimension. In the simplest case of a 5D space-time the warping is introduced to define a hierarchy of energy scales. Thus, gravity, which is distributed in both the bulk and on the brane can have the Planck energy, while ordinary physics, whose particles and interactions are localized only on the brane, can be characterized by a much lower energies.

The idea of space-time warping extends to a greater number of additional compact dimensions $(6$, in the case of a $10 \mathrm{D}$ space-time). In the simplest models, the extra dimensions are spheres or tori, characterized by $A(y)=$ const. There are more complicated models where warp factor $e^{-A(y)}$ strongly depends on the coordinate $y$. Thus, in the so-called throat of multi-dimensional space-time, this function is small, and far away from the throat is close to unity. For an observer located in the $4 \mathrm{D}$ space-time it means that the observable tension (or linear density) of the cosmological fundamental strings is $\mu_{0}=e^{-A(y)} \cdot \mu$, where $\mu$ is defined in $10 \mathrm{D}$ space-time. In other words, $\mu_{0}$ comes from real observational data (from the CMB $\delta T / T$ upper limits or from the statistically averaged optical data $\Delta \Theta)$, and $\mu$ is theoretical property of the superstring.

Therefore, the observable energy of the fundamental string could be much smaller than its energy in 10D space-time.

It is important to note that in the brane-world scenario together with the birth of cosmological superstrings it could naturally be realized the inflation stage of the early universe (see [23]). Inflation 
occurs at a low value of the function $e^{-A(y)}$. Inflation is carried out in the process of annihilation of a brane and an anti-brane. In this process branes and anti-branes of higher dimensions can produce branes of lower dimensions, including the fundamental strings. The string energy scale is not Planck one, it is much lower because the strings are created in areas of high warping. Thus, the inflation energy is directly linked with the energy of fundamental superstrings (being in frames of the brane-world scenario).

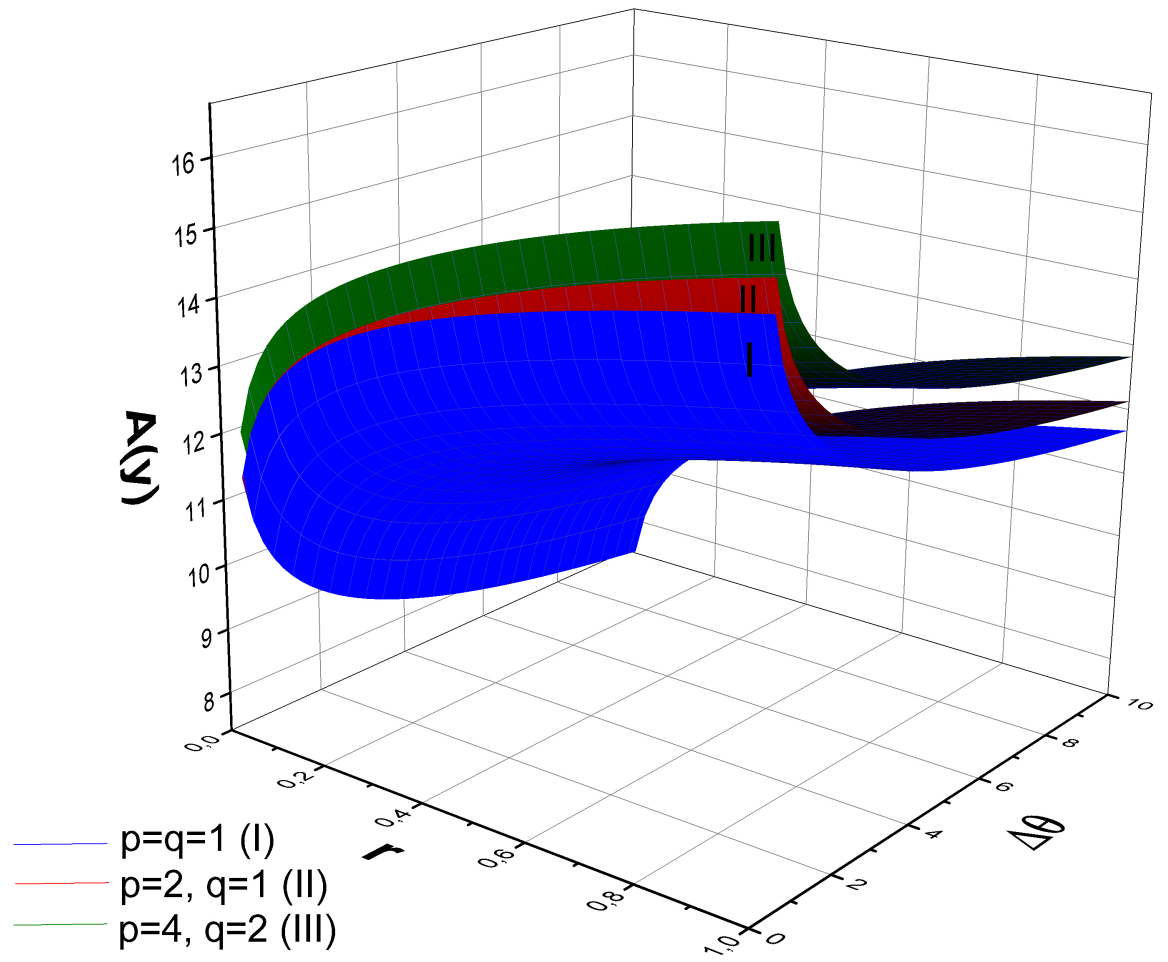

Figure 1. The 3D diagram of the dependence of the warp factor function $A(y)$ on the deficit angle $\Delta \Theta$, measured in arcsec, and on the ratio of the Planck length to the length of the fundamental string $r=l_{p} / l_{s}$ is presented. The different colors correspond to different values of $p, q$, describing the mixed state configuration of the string, consisting of $p F$-strings and $q D$-strings. For inflation models on the brane with the warp factor of the order of $10^{-4},[23]$, the energetically preferred configuration are those with the least number of the $F$ - and $D$-strings.

\section{Connection between 4D Cosmological Superstrings and 10D Superstrings}

The tension of the fundamental strings, observable in the usual $4 \mathrm{D}$ space-time, is related to its $10 \mathrm{D}$ tension proportionally to the warp factor, [25]

$$
\mu_{0}=e^{-A(y)} \cdot \mu_{p, q}^{(10)} \text {. }
$$

Indices $p$ and $q$ represent the number of F- and D-strings respectively. As F-, and D-strings can be generated during inflation in the annihilation process of branes and anti-branes. F- and D-strings can combine to form one-dimensional cosmological objects called FD-strings (as visual analogy for the case, for example, $p=1, q=1$ there could be two lines approaching each other at a certain angle, and then twisted with one another into a single thread).

The cosmic string tension from the point of view of $4 \mathrm{D}$ observer is related with the string velocity and with the temperature amplitude of the CMB, [26], [27]

$$
\frac{\delta T}{T}=\frac{8 \pi G \mu_{0}}{c^{2}} \cdot \frac{\beta}{\sqrt{1-\beta^{2}}} .
$$


Here $\beta$ is the component of cosmic string velocity, normalized to the speed of light, perpendicular to the line of sight, $T=2.73$ is the temperature of the $\mathrm{CMB}, \delta T=40 \mu \mathrm{K}$ is the observational limit on the maximal value of the CMB anisotropy generated by a single cosmic string, obtained by MHF method [15].

For example in the simplest case of Nambu-Goto network simulations the string velocity is fixed: $\beta=1 / \sqrt{2}([15],[28])$. It is important to note that now the ability to search individual strings in the CMB anisotropy data provides the ability to constrain the parameter space allowed for $\beta$ and linear density $\mu_{0}$ of a string as a function of the upper limit on the anisotropy $\delta T / T=F\left(\beta, \mu_{0}\right)$.

On the other hand, one can use observational data of a completely different nature, from optical surveys. Rewriting the expression for linear density in the Planck unit system $(\hbar=c=1)$

$$
\mu_{0}=\Delta \Theta \frac{1}{8 \pi} \cdot \frac{1}{l_{p}^{2}}
$$

where $l_{p}=\sqrt{G}$ is the Planck length, and $\Delta \Theta=8 \pi G \mu_{0} / c^{2}$ is the string deficit angle, which is the angle of the 3D space of the cone, which replaces the 3D Euclidean space of our universe in the presence of a cosmic string, [3]. From the point of view an observer the deficit angle is proportional (in the case of large cosmic string distance from the observer, asymptotically equal) to the angular distance between the components of pairs of gravitationally lensed images of distant galaxies lensed by a cosmic string. Therefore, in the approach of model the remote string $\Delta \Theta$ is observational parameter, which should be estimated statistically analyzing the chain of gravitational lenses along the path of the string.

Obviously, at different velocities $\beta$ with the same magnitude of the anisotropy the deficit angle should be different. Using both the radio and optical data we can reconstruct the more probably string velocity as $\langle\beta\rangle=\tilde{F}(\Delta \Theta, \delta T / T)$, where in general $\Delta \Theta$ should be a statistical value coming from observational data,

$$
\Delta \Theta=\left\langle\Delta \Theta> \pm \frac{\sigma_{\text {lens }}}{\sqrt{N_{\text {lens }}}} .\right.
$$

Here $N_{\text {lens }}$ is the number of gravitational lenses along the proposed (by CMB data) position of a string and $\sigma_{\text {lens }}$ is the standard deviation of the measurement of the distances between the pairs. It will be done in future work (the analysis of gravitational lenses along the cosmic string candidate with $\delta T / T=40 \mu K$, [15], now in progress).

Using the string velocity derived from the string network simulations, [29], [30], and observational constraint on string anisotropy, we can estimate the theoretically expected deficit angle (we summarize the results in Table (1)).

Table 1. There are presented the values $\beta$, obtained by simulation of the dynamics of string networks (there are listed the authors of the respective papers). From the values of $\beta$ is calculated the deficit angle (in arcsec), which corresponds to the anisotropy of $40 \mu K$ (see text). The first and last columns of pairs $\beta$ and $\Delta \Theta$ are presented as limiting values that do not contradict the observational data.

\begin{tabular}{lllllll}
\hline Properties/references & & $\begin{array}{l}{[29]} \\
\text { radiation ep. }\end{array}$ & $\begin{array}{l}{[30]} \\
\text { radiation ep. }\end{array}$ & $\begin{array}{l}{[29]} \\
\text { matter ep. }\end{array}$ & $\begin{array}{l}{[30]} \\
\text { matter ep. }\end{array}$ \\
\hline$\beta$ & 0.99 & 0.66 & 0.62 & 0.61 & 0.58 & 0.50 \\
$\Delta \Theta\left({ }^{\prime \prime}\right)$ & 0.43 & 3.47 & 3.86 & 3.97 & 4.29 & 5.29 \\
\hline
\end{tabular}

Now let us turn to (3). The 10D tension is expressed by the formula, [25]

$$
\mu_{p, q}^{(10)}=\frac{1}{2 \pi l_{s}^{2}} \cdot \sqrt{p^{2}+\frac{q^{2}}{g_{s}^{2}}},
$$

where $g_{s}$ is the string coupling constant, $l_{s}$ is the length of the fundamental string (defined by its creation energy). 


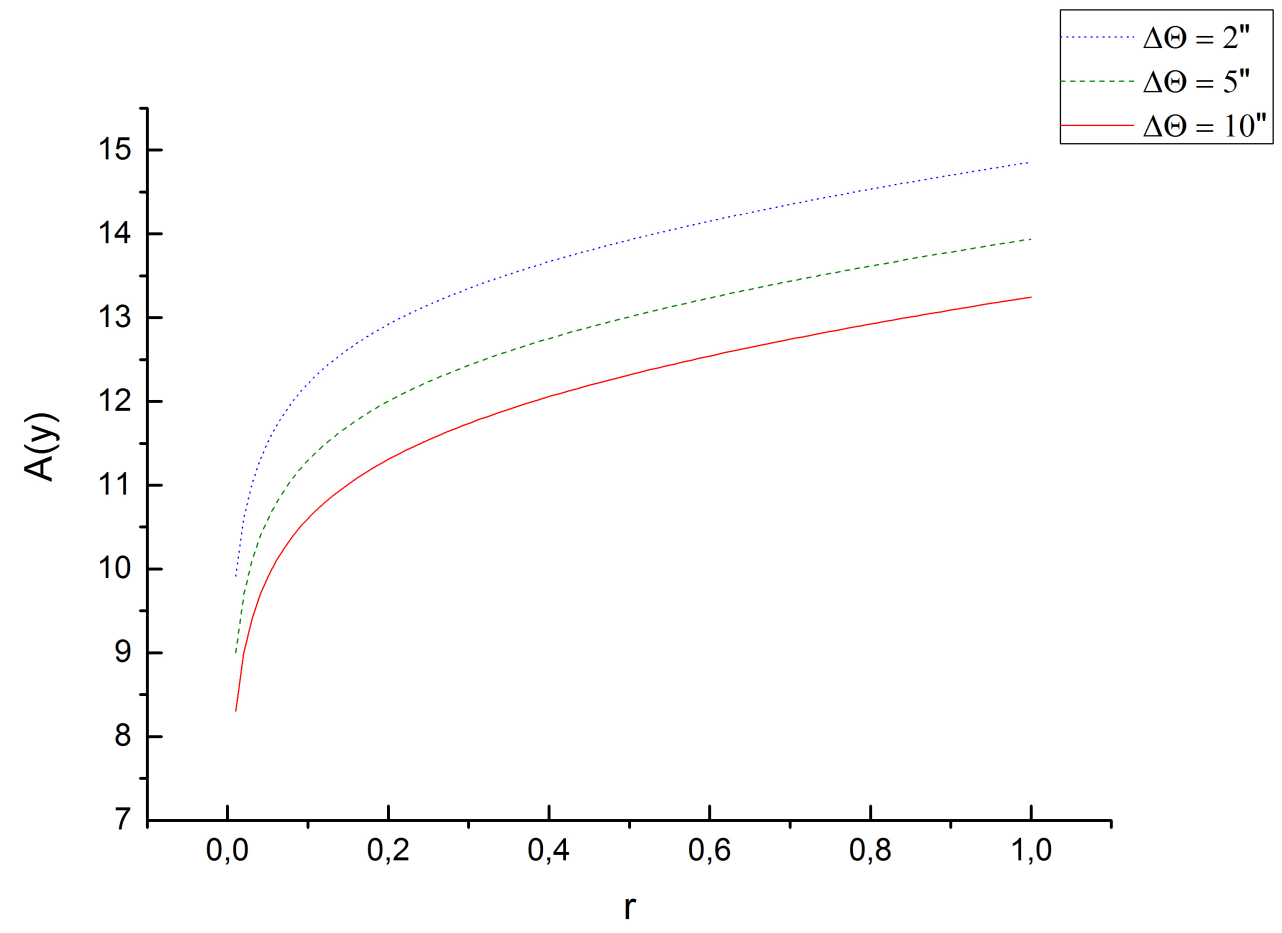

Figure 2. The dependence of the warp factor function $A(y)$ (see (10)) of the additional dimension from the ratio of the Planck length to the length of the fundamental string $r=l_{p} / l_{s}$ (cross section at different $\Delta \Theta$ of the surfaces in Fig. (1) for $p=q=1$ ). When the length of the string is much larger than the Planck (model of large extra dimensions), fundamental strings are created during the inflation stage, which corresponds to the warp factor of the order of $10^{-4}$ or $A(y) \approx 9$. The values of the warp factor are significantly sensitive to observational parameter of a string - deficit angle.

In the 10D string theory, the coupling constant $g_{s}$ can be expressed in terms of the length of fundamental string and Planck length and also through the $4 \mathrm{D}$ Newton gravitational constant $G=g_{s}^{2} \alpha^{\prime}, l_{s}=\sqrt{\alpha^{\prime}}, g_{s}^{2}=$ $G / \alpha^{\prime}=G / l_{s}^{2}=l_{p}^{2} / l_{s}^{2}$, where $\alpha^{\prime}$ is fundamental string parameter, which is called inclination angle, $\left[E^{-2}\right]$.

Let us suppose that from optical observation we obtained the value $\langle\Delta \Theta\rangle$ and calculated $\sigma_{\text {lens }}$ as we discussed above. Then, substituting in (3) expressions (7) and (5)

$$
\Delta \Theta \cdot \frac{1}{8 \pi} \cdot \frac{1}{l_{p}^{2}}=e^{-A(y)} \cdot \frac{1}{2 \pi} \cdot \frac{1}{l_{s}^{2}} \cdot \sqrt{p^{2}+q^{2} \frac{l_{s}^{2}}{l_{p}^{2}}} .
$$

On the left part of the equation (8) there is the observable deficit angle (derived from the observed gravitational lensing events). On the right part of the equation (8) there is a function which depends on the FD-string energy. Thus the FD-string energy is constrained by observable quantities, i.e. the deficit angle. This gives us the ability to link the FD-string energy with the string properties observable by astronomical methods.

Transforming the (8), we obtain:

$$
e^{A(y)}=\frac{4}{\Delta \Theta} \cdot\left(\frac{l_{p}}{l_{s}}\right)^{2} \cdot \sqrt{p^{2}+q^{2}\left(\frac{l_{s}}{l_{p}}\right)^{2}} .
$$

The warp factor function $A(y)$ from (9) is

$$
A(y)=\ln \left(\frac{4 r^{2} \sqrt{p^{2}+q^{2} / r^{2}}}{\Delta \Theta}\right),
$$


where $r=l_{p} / l_{s}$ is the ratio of fundamental string length to the Planck length.

In the Fig. (1) the warp factor function $A(y)$ as a function of the string deficit angle $\Delta \Theta$ (in arcsec) and of the ratio of the Planck length to the length of the fundamental string $r=l_{p} / l_{s}$ is presented. The different colors correspond to different values of $p, q$, describing the mixed state configuration of the string, consisting of $p F$-strings and $q D$-strings. For inflation models on the brane with the warp factor of the order of $10^{-4},[23]$, the energetically preferred configuration are those with the least possible number of the $F$ - and $D$-strings, $p=q=1$.

If we fix the number of $F$ - and $D$-strings with their more reliable values, i.e. $p=q=1$, we can see that the warp factor function $A(y)$ is significantly sensitive to observable string parameter (deficit angle), see Fig. (2) where the dependence of the function $A(y)$ from the ratio of the Planck length to the length of the fundamental string $r=l_{p} / l_{s}$ is presented for different values of the deficit angle $\Delta \Theta$. When the length of the string is much larger than the Planck (model of large extra dimensions), fundamental strings are created during the inflation stage, which corresponds to the warp factor of the order of $10^{-4}$ or $A(y) \approx 9$.

From the common assumption about the existence of extra dimensions, the function $A(y)>0$, therefore, $r>r_{\text {crit }}$

$$
r_{\text {crit }}=\frac{1}{\sqrt{2} p} \cdot \sqrt{\sqrt{q^{4}+p^{2}\left(\frac{\Delta \Theta}{2}\right)^{2}}-q^{2}} .
$$

Since the value $(p \cdot \Delta \Theta / 2)<<1$, up to the third order $r_{c r i t}=\Delta \Theta / 4 q$.

Therefore, $l_{p} / l_{s}>\Delta \Theta / 4 q$ or

$$
\frac{m_{s}}{m_{p}}>\frac{\Delta \Theta}{4 q} .
$$

Substituting into (12) the Planck mass (in GeV) we obtained the minimal possible energy of FD-string is given by right side of an inequality

$$
m_{s}>\frac{\Delta \Theta}{4 q} \cdot 1.2 \cdot 10^{19}
$$

Normalizing in (13) the deficit angle $\Delta \Theta$ on the astronomical units (arcsec) we finally have

$$
m_{s}>\left(\frac{\Delta \Theta}{2^{\prime \prime}}\right) \cdot \frac{2.88 \cdot 10^{13}}{q} .
$$

Note that the resulting restriction does not depend on the number of F-strings.

The tension $G \mu_{s}$ recalculated from the string energy (14) is consistent with those of other authors $\left(10^{-12}<G \mu_{s}<10^{-6},[31],[32]\right)$, and refines this limitation depending on observable deficit angle. So, for example, D-string tension $G \mu_{s}=m_{s}^{2} /(4 \alpha \pi)$, where $\alpha \approx 1 / 25$ is the gauge coupling, [31]. For our case (14)

$$
G \mu_{s}>\left(\frac{\Delta \Theta}{2^{\prime \prime}}\right)^{2} \cdot \frac{10^{-11}}{q^{2}} .
$$

For large, but acceptable by observations, deficit angle $\Delta \Theta=5.29^{\prime \prime}$ (which corresponds to string velocity $\beta=0.5$, see Table (1)) minimum value of the tension reaches a value of about $6.9 \cdot 10^{-11}(q=1)$.

For strings, moving with about light velocity $\beta=0.99$, the minimum value of tension becomes of the order $10^{-13}$. So, lower string velocities are more in agreement with the previous estimation from brane-inflation models, [31].

Note also that all previously obtained estimations of cosmic string linear density were based on the cumulative assessment of the possible contribution of the spectrum of cosmic strings in the spectrum of the CMB anisotropy, while our estimates are based on an analysis of individual candidates for cosmic strings.

Because of the quadratic dependence from $q$ with the growth of this parameter the lower limit in (15) obviously decreases, but is insensitive to the number of F-strings. So, from observational point of view for bound state of FD-string it is possible to distinguish between contribution one D-string and many.

As it was mentioned above (see. Table 1), for a given value of $\delta T / T$ (which has been defined by analysis of CMB anisotropy data, [15]) there is a unique relationship between the string velocity $\beta$ and 
deficit angle $\Delta \Theta$. For classical strings (not superstrings) definition by independent methods (for example, by searching for strings of gravitational lens pairs of distant galaxies) of the string deficit angle allows to uniquely constrain its velocity, and its tension $\mu$. In the case of superstrings by knowing the deficit angle, one could restore the warp factor $e^{-A(y)}$ and determine the creation energy of the strings.

\section{Conclusions}

If the brane-world scenario is correct, then the annihilation of the brane and antibrane creates FD-strings. Using the $4 \mathrm{D}$ observational features of the possible string candidates one should put a lower limit on the superstring linear density $\mu_{s}$

$$
G \mu_{s}>\left(\frac{\Delta \Theta}{2^{\prime \prime}}\right)^{2} \cdot \frac{10^{-11}}{q^{2}} .
$$

where $q$ is the number of D-strings and $\Delta \Theta$ is the string deficit angle (in arcsec). This limit is derived from the simple condition of positiveness of the warp factor function $A(y)$ in the warp space-time with the metric

$$
d s^{2}=e^{-A(y)}\left(d t^{2}-d x^{2}\right)-d y^{2} .
$$

Due to new projects on gravitational lensing studies and the CMB data analysis some energy bands for cosmic strings to exist are closing, [16]. In addition, it is less hope to observe so-called "light" strings (with energies less than $10^{15} \mathrm{GeV}$ ) in CMB data, [8].

However, some new possibilities for strings are opening. The cosmological superstring could be characterized through astrophysically measurable quantities (the string deficit angle).

The fixed value of CMB anisotropy induced by a string and known deficit angle (by gravitation lensing data) could uniquely restore the string velocity and string linear density. In the case of cosmological superstring one could restore the warp factor and determine the string creation energy.

Acknowledgments. The authors thank Prof. M.V. Sazhin for useful discussions.

O.S. thanks the Department of Mathematical and Physical Sciences of the The University of Campania Luigi Vanvitelli for the hospitality.

\section{References}

1. A. Vilenkin, "Gravitational field of vacuum domain walls and strings," Phys.Rev.D 23 4, p. 852, 1981.

2. —_ "Cosmic strings as gravitational lenses," Ap. J. L51 282, p. 282, 1984.

3. E. Shellard and A. Vilenkin, "Cosmic strings and other topological defects," Cambridge Univ.Press, UK, 1994.

4. A. Vilenkin, "Looking for cosmic strings," Nature 322, p. 613, 1986.

5. Y. Zeldovich, "Cosmological fluctuations produced near a singularity," MNRAS 192, p. 663, 1980.

6. T. Kibble, "Topology of cosmic domains and strings," J.Phys.A:Math.Gen., p. 9, 1976.

7. M. Hindmarsh and T. Kibble, "Cosmic strings," Rept.Prog.Phys.58, pp. 477-562, 1995. [Online]. Available: arXiv:hep-ph/9411342

8. P. C. Ade, N. Aghanim, C. Armitage-Caplan, and et al., "Planck 2013 results. xxv. searches for cosmic strings and other topological defects," A\&A, pp. 571, A25, 2014. [Online]. Available: arXiv:1303.5085

9. T. W. B. Kibble and T. Vachaspati, "Monopoles on strings," J. Phys. G: Nucl. Part. Phys. 42 094002, 2015. [Online]. Available: arXiv:1506.02022

10. A.-C. Davis and T. Kibble, "Fundamental cosmic strings," Contemp.Phys. 46, pp. 313-322, 2005. [Online]. Available: arXiv:hep-th/0505050

11. G. R. Dvali and S. H. H. Tye, "Brane inflation," Phys. Lett. B450, p. 72, 1999. [Online]. Available: arXiv:hep-ph/9812483

12. C. P. Burgess, M. Majumdar, D. Nolte, F. Quevedo, G. Rajesh, and R.-J. Zhang, "The inflationary brane-antibrane universe," JHEP 07, p. 47, 2001. [Online]. Available: arXiv:hep-th/0105204

13. M. Majumdar and A. Christine-Davis, "Cosmological creation of d-branes and anti-d-branes," JHEP 03, p. 56, 2002. [Online]. Available: arXiv:hep-th/0202148

14. R. J. Danos and R. H. Brandenberger, "Canny algorithm, cosmic strings and the cosmic microwave background," Int.J.Mod.Phys. D19, p. 183, 2010. [Online]. Available: arXiv:0811.2004 
15. O. S. Sazhina, D. Scognamiglio, and M. Sazhin, "Observational constraints on the types of cosmic strings," The European Physical Journal C 74:2972, 2014. [Online]. Available: arXiv:1312.6106

16. E. Morganson, P. Marshall, and et al., "Direct observation of cosmic strings via their strong gravitational lensing effect: Ii. results from the hst/acs image archive." [Online]. Available: arXiv:0908.0602v1

17. M. Sazhin and et al., "Gravitational lensing by cosmic strings: what we learn from the csl-1 case," $M N R A S$ 376, p. 1731, 2007.

18. — " Csl-1: chance projection effect or serendipitous discovery of a gravitational lens induced by a cosmic string?" MNRAS 343, p. 353, 2003.

19. P. Bhattacharjee and G. Sigl, Phys. Rep. 327, p. 109, 2000.

20. T. Vachaspati, Phys. Rev. D 81043531 1, 2010.

21. R. Caldwell, R. Battye, and E. Shellard, Phys. Rev. D54, 7146, 1996.

22. C. Corda, "Interferometric detection of gravitational waves: the definitive test for general relativity," Int. J. Mod. Phys. D 18, 2275, 2009. [Online]. Available: arXiv:0905.2502v3

23. J. Polchinski, "Introduction to cosmic f- and d-strings." [Online]. Available: arXiv:hep-th/0412244

24. V. A. Rubakov, "Large and infinite extra dimensions," Phys. Usp. 44, pp. 871-893, 2001.

25. Schwarz and J. An, "Sl(2,z) multiple of type iib superstrings," Phys. Lett. B 360, p. 13, 1995.

26. N. Kaiser and A. Stebbins, "Microwave anisotropy due to cosmic strings," Nature 310, pp. 391-393, 1984.

27. O. Sazhina, M. Sazhin, V. Sementsov, M. Capaccioli, G. Longo, G. Riccio, and G. D'Angelo, "Cmb anisotropy induced by a moving straight cosmic string," Journal of Experimental and Theoretical Physics 106, 5, pp. 878-887, 2008.

28. E. Copeland and T. Kibble, "Cosmic strings and superstrings," Proc. Roy. Soc. Lond. A 466, 623657, 2010. [Online]. Available: arXiv:0911.1345v3

29. D. Bennet and F. Bouchet, "High resolution simulations of cosmic string evolution: network evolution," Phys. Rev. D41, p. 2408, 1990.

30. B. Allen and E. Shellard, "Cosmic string evolution - a numerical simulation," Phys. Rev. Lett. 64, p. 119, 1990.

31. S. H. H. Sarangi, S.and Tye, "Cosmic string production towards the end of brane inflation," Phys. Lett. B536, p. 185, 2002. [Online]. Available: arXiv:hep-th/0204074

32. H. Jones, N. T.and Stoica and S. H. H. Tye, "Brane inflation and semilocal strings," Phys. Lett. B563, p. 6, 2003. [Online]. Available: arXiv:hep-th/0303269 